\title{
Research on Flashover Breakdowns and Prevention Measures of Synthetic Insulator of overhead Power Transmission Line
}

\author{
Yi Guoqi, Jia Yang, Huang Yujun, Yu Jintao \\ Inspection and maintenance Company, Power Supply Company of Xinyang, Xinyang, Henan, \\ China
}

guoqi123@163.com

\begin{abstract}
Keywords: breakdowns and prevention measures; synthetic insulator; overhead power transmission line
\end{abstract}

\begin{abstract}
As an anti-pollution flashoverfacility, synthetic insulator is now widely used in the overhead transmission lines in China. It has good mechanical properties, nice anti-pollution flashover performance, strong electrical erosion resistance and high efficiency advantages in operation. However, the lightning, the bird, icing, filth, foreign body and other unknown reasons of flashover fault will influent the safety of overhead transmission lines. Therefore, it is important to improve the safety operation of the overhead transmission line.
\end{abstract}

\section{Structure of Synthetic Insulator}

Synthetic insulator shape structure is similar to the suspension insulator, including the end fittings, insulation umbrella bellows, bellows and can heart bar. Also known as composite insulators for organic composite insulator, rubber insulator and porcelain insulator, it is by organic synthetic material to form a composite insulator, mainly mandrel, fittings and umbrella skirt sheath and adhesive layer. Fittings is the mechanical loading of the composite insulator of a transmission member, it and the mandrel assembled together to form a composite insulator of the connector. The structure affects the strength of the mandrel play and the mechanical properties of insulator. Umbrella skirt sheath is synthetic insulator insulating parts. Its role is the composite insulators with high enough resistance to wet flashover and pollution flashover of insulation performance, mandrel protection from atmospheric corrosion. The mandrel is bearing components of composite insulators mechanical load, but also is the main component of internal insulation. Bonding layer part is with the adhesive between the sheath and the plug connections; another part is with potting to complete the connection between the umbrella skirts. The quality of the adhesive is directly related to the level of the internal insulation.

\section{Characteristics of Synthetic Insulator}

\section{Advantages}

Synthetic insulator core rod consists of epoxy glass fiber thermal mixing extrusion and expansion of its strength is 1.5 times than that of ordinary steel and high strength porcelain 3-4 times than axial tension. It also has a strong vibration absorbing ability.Seismic damping performance is very high. This shows that the synthetic insulator mechanical performance superiority manifests itself only in the core itself. Synthetic insulator has a unique hydrophobic performance, in the rain, the wet umbrella insulator corrugated surface does not form a water film, but a drip - drip, to the surface to form a conductive channel, the pollution flashover voltage is higher, is 3 times than that of the same voltage grade porcelain insulator, suitable for used in overhead transmission line in heavy pollution area. However, after a certain number of years of operation, by the role of long-term ultraviolet and electric field, hydrophobic properties will decline year by year, in a long time of rainy days, the water is not easy to recover. Therefore, the synthetic insulator which operates normally for many years sometimes will get unknown causes flashover. 


\section{Disadvantages}

Synthetic insulators are more expensive than porcelain insulator in its raw materialsand production process. Its umbrella skirt is made of silicon rubber. The relatively soft material, vulnerable to injury and damage the seal, resulting in decreased insulation properties. Due to organic synthesis insulator silicon rubber, although adding anti-aging agent, glass, porcelain inorganic insulator life than the short. Therefore, synthetic insulator is going to be aging after some years in the nature environment.

\section{Prevention Measures of Flashover Breakdowns of Synthetic Insulator}

\section{Lightening Flashover and Prevention Measures}

Overhead transmission lines always operate in the wild, the path through the section of the operating environment has different, a large part in region. Under special weather conditions, frequent the lightning invasion, due to the same voltage rating of overhead transmission lines, synthetic insulation has enough dry arc distance is to ensure that their home should be lightning withstand level of important factors. We analyze the synthetic insulator in Xinyang city linked to network operationvast majority of lightning flashover is $110 \mathrm{kV}$ transmission line, which most of synthetic insulator effectively dry arc distance does not meet the $1000 \mathrm{~mm}$, far less than the IEC about the requirements of the standard insulators $1050 \mathrm{~mm}$. It can be seen that the dry arc distance is small and the standard is the main reason for the lightning flashover of composite insulators. At the same time, the electric field distribution of composite insulator is the main reason of the failure of the composite insulator.

Preventive measures: In the preliminary design of new overhead transmission line, we should according to the area of the flash density and comprehensive analysis to determine region and consider avoiding the path of the line to. If you really can't avoid the thunderstorm area, according to the different voltage grade line insulation configuration, appropriate adjustment of synthetic insulator dry arc length, installation of pressure ring and improve the synthetic insulator electric field distribution to solve. Also according to the different sections of a mountain and multiplexers can also be taken to install the line surge arrester or controllable discharge lightning needle differentiation and lightning protection measures to avoid lightning overhead transmission lines on the insulation configuration consisting of a direct threat. We should reinforce the synthetic insulators daily technical supervision to prevent the flashovers.

\section{Contamination Flashover and Prevention Measures}

The new synthetic insulator has good anti-pollution flashover ability. But the heavier dust and other dirt falling on the insulator surface, under the combined effect of mechanical force thousands of minor surface corrosion, rough degree increase, form invisible tiny cracks, attached to the surface of the contamination layerwith the overhead transmission line in the long run. In bad weather, such as fog, dew, high humidity weather, rain can be to form water droplets on the surface of the insulator, in the serious surface corrosion and contamination layer combined sewage droplet formation, due to the impurity layer of uneven distribution and moist the insulator surface produce local high pressure part, which occurred in the point like discharges consumed small water droplets surrounding polymer layer, damage of silicone rubber hydrophobicity, sometimes even a temporary loss of hydrophobicity, so the years of operation of composite insulators, under certain climatic conditions, especially high humidity, low temperature climate environment, sometimes with pollution flashover fault.

Preventive measures: We should increase the synthetic insulator sampling efforts. Organizations live working samples of happened synthetic insulator accident spot radius of $5 \mathrm{~km}$ within the tower, according to the insulator installation phase cycle sampling. Height insulator surface color, charged hydrophobic monitoring using instrument and part dirt and synthetic insulator near the water's take charged replacement

\section{Bird Excrement Flashover and Prevention Measures}

Synthetic insulator flashover may be caused by bird droppings. According to statistics for 2013, the synthetic insulator accident lightning flashover accidents accounted for 37\%, unidentified 
flashover accidents accounted for $23 \%$, only confirm the guano flashover accidents accounted for $16 \%$. Guano flashover accident occurred mostly in the $110 \mathrm{kV}$ and $220 \mathrm{kV}$ transmission line, $35 \mathrm{kV}$ and below and $500 \mathrm{kV}$ power. On the one hand due to the increase of the grid voltage, the length of the air gap which may cause flashover is also increased. The maximum operating voltage of $73 \mathrm{kV}$ $110 \mathrm{kV}$ phase, may lead to the long air gap flashover reaching about $15 \mathrm{~cm}$. On the other hand, because of the insulator insulation distance is not very long; guano channel can be continuously across the length. $110 \mathrm{kV}$ and $220 \mathrm{kV}$ insulator is widely used are pressure ring, guano along the voltage outer ring and close to the voltage drop ring, led directly to the upper and lower hardware, pressure ring between the electrical short circuit discharge, insulator flashover is leaving no guano traces, flashover mechanism is dropping instantaneous distortion around the insulator electric field distribution. The bird excrementchannel and high voltage insulator pressure equalizing ring or fittings between the air gap breakdowns will cause flashovers. It is shown in Figure 1 as follows:

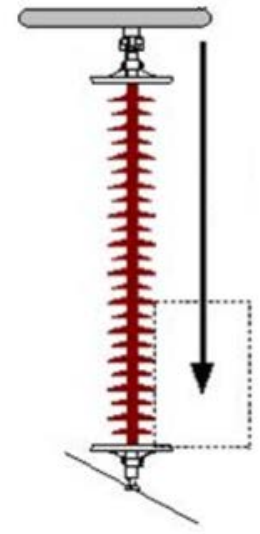

Figure 1 bird excrement flashover

Preventive measures: We should prevent bird activity on the tower. On line tower installation bird driving device, scare and drive away birds close to the tower and on the tower is easy to cause the fault position to stay; prevent birds on the tower of key parts, in the insulator string above the installation of protective measures, such as baffle, anti-bird thorn or switch to high along the plate porcelain insulator to prevent birds on the insulator string is the top perches defecation. To do a good job for overhead transmission line bird damage statistical analysis, including statistical analysis bird of the geographical and climatic characteristics, the bird occurrence time, bird damage to the tower, insulator types and voltage levels, caused by failure of the birds and so on and according to the characteristics of the region take comprehensive treatment of differentiated bird proof. That is to say, we should take across the board installation bird proof sting, the device of driving birds, bird key sections of the installation of anti-bird thorn, bird or comprehensive treatment measures against bird sealing box (Figure 2).
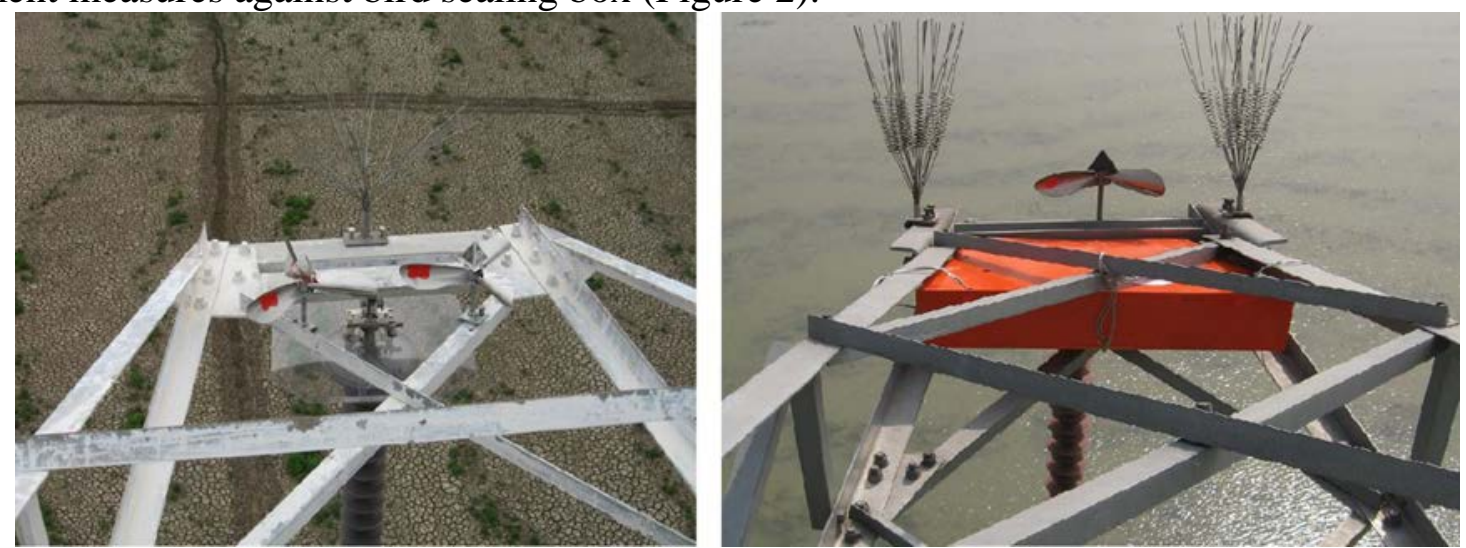

Figure 2 bird excrement flashover prevention measures 


\section{Foreign Body Flashover and Prevention Measures}

As the overhead transmission lines are disposed in the high winds environment, such as bad weather encountered, near the channel line of plastic film, the kite line, aluminum foil, plastic rope and other foreign body volume and wound on the surface of the composite insulators. At this time if such foreign matter entrained metal wire or dirty, will be short insulator effectively climb electrical distance, similarly, distortion of the synthetic insulator around the electric field distribution. The synthetic insulator which is in the foreign body flashover is similar to the bird excrement flashover. (Figure 3)
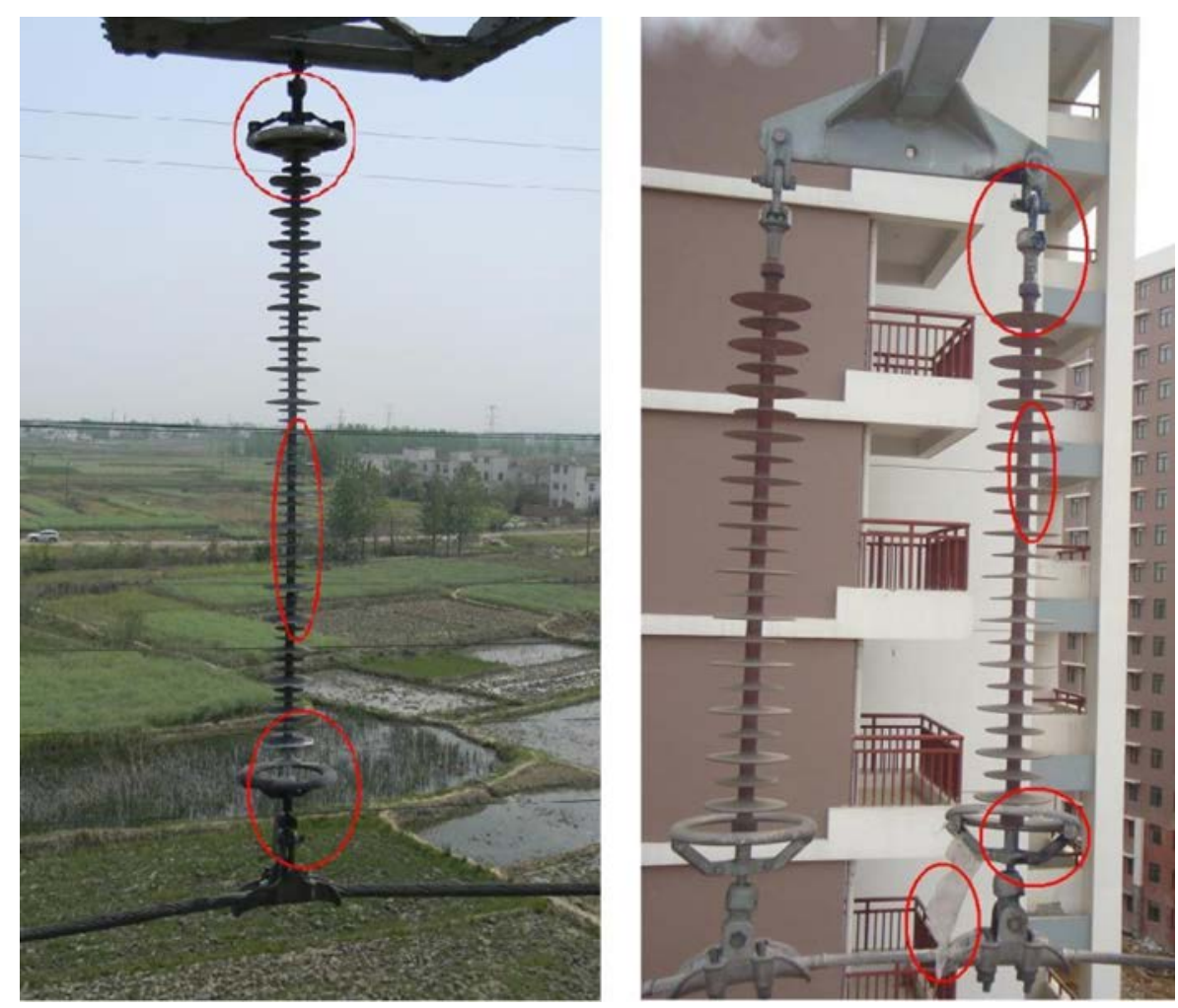

Figure 3 comparisons between bird excrement flashover and foreign body flashover

Preventive measures: In the design of overhead transmission lines, we should take the foreign body of hazards in key periods for statistical, before the special weather comes to carry out publicity on the protection of power facilities. For the large area plastic greenhouse can take the initiative to helping reinforcement; for the holidays kite regional locations and rely on the local government functional departments collaborative discouraged and stop. At the same time, it is essential to do a good job in the status of the line channel inspection work to avoid the foreign body flashovers.

\section{References}

[1] Shi Shuaijun, The Reason and Presentation Research of Synthetic Insulator Flashing over under Working Condition, D. Chongqing University. 2008.

[2] Li Zhengliang, The Performance of Pollution Flashover and UV Imaging Characteristics of Composite Insulator, D. Chongqing University. 2005.

[3] Zhang Gangqiang, Overhead Power Transmission Lines Huge Market Potential for Copper and Aluminum Industry, J. China Nonferrous Metals Monthly. 9(2012) 34- 36. 